\title{
Geleitwort der
}

\section{Gründungsherausgeber_innen}

Vor 10 Jahren - also im Jahr 2011 - erschien die erste Ausgabe der Zeitschrift Frühe Bildung. Im ersten Editorial haben wir Gründungsherausgeber_innen damals dargelegt, warum wir es für angebracht hielten, diese neue Zeitschrift ins Leben zu rufen. Insgesamt hatten wir vier Gründe benannt. Ein erster Grund bestand in der Feststellung, dass Überzeugungen und Realitäten zur Qualität von Bildung nicht zwangsläufig deckungsgleich sind. Wir wollten folglich den faktenbasierten Diskurs im Feld der frühen Bildung ankurbeln.

Ein zweiter Grund war die gemeinsame Einschätzung, dass wesentliche und entscheidenden Weichen für die Qualität und den individuellen Erfolg in unserem Bildungssystem bereits vor Vollendung des ersten Lebensjahrzehnts gestellt werden. Wir rechneten daher mit vermehrtem - auch politisch gewolltem - Forschungsbedarf in der frühen Bildung.

Drittens wollten wir ein Forum für den Diskurs unter den verschiedenen wissenschaftlichen Disziplinen schaffen, die sich mit den Bildungsprozessen in Kindertageseinrichtungen und im Schulanfangsunterricht beschäftigen.

Viertens sahen wir angesichts der damals an vielen Orten neu eingerichteten Studiengänge zur frühen Bildung an Hochschulen den Bedarf für ein Format des Austausches zwischen Wissenschaft und Bildungspraxis zu Themen der frühen Bildung einschließlich des Schulübergangs und der Schuleingangsstufe.

Die Zeitschrift sollte sich an Wissenschaftler_innen sowie an Dozent_innen wenden, die in Hochschulen, Fachschulen und Weiterbildungen frühpädagogische Fachkräfte ausbilden und beraten, außerdem auch an Studierende sowie an fachwissenschaftlich interessierte Mitarbeiter innen in Jugendämtern, Kindertageseinrichtungen, Trägerinstitutionen und Grundschulen.

Die Gründung der Frühen Bildung fiel in eine Zeit des Optimismus und Aufbruchs: Die Bedeutsamkeit der frühen Jahre für die Persönlichkeitsentwicklung und Bildungsbiografie wurde zunehmend auch außerhalb von Fachkreisen erkannt. Kindertageseinrichtungen wurden als einflussreiche gesellschaftliche Institutionen der Bildung und Erziehung junger Kinder wahrgenommen. Bundesweit entstanden Studiengänge zur akademischen
Qualifizierung kindheitspädagogischer Expertinnen und Experten. In diesem Klima konnte sich die Frühe Bildung erfolgreich als eine wissenschaftliche, stark forschungsorientierte Fachzeitschrift etablieren, die empirische Befunde zu zentralen Themen und Problemen des frühpädagogischen Feldes vorstellt sowie Diskurse in Wissenschaft und Praxis aufgreift.

10 Jahre sind seither vergangen. Auch wenn nicht alle Hoffnungen erfüllt und Ziele erreicht wurden, haben wir den Eindruck, Vieles, von dem mit der Zeitschrift erreicht zu haben, was uns vorschwebte. So ist es gelungen, mit der Frühen Bildung eine Fachzeitschrift mit wissenschaftlichen Standards zu etablieren, die nicht nur die Methodenvielfalt der früh- und kindheitspädagogischen Forschung widerspiegelt, sondern in der in ausgewogener Form auch die Kerndisziplinen (Früh-)Pädagogik und (Entwicklungs-) Psychologie repräsentiert sind. Auch sehen wir es als gelungen an, über die Jahre hinweg die zentralen Themen des Fachdiskurses - von der Qualitätsentwicklung auf organisationaler Ebene bis hin zur Förderung und Begleitung von Kindern in unterschiedlichen Entwicklungsfeldern - abzubilden. Zugleich besteht „Luft nach oben“: Die Analyse der Abonnent_innen zeigt, dass der Verbreitungsgrad vor allem bei den für Fort- und Weiterbildung Verantwortlichen, den Fachberatungen und Lehrkräfte an Fachschulen noch zu wünschen übriglässt.

Wir hatten mit dem Start der Zeitschrift auch die Hoffnung verbunden, allen empirischen Forschungsansätzen im Feld ein Forum zu bieten, insbesondere auch den qualitativen und rekonstruktiven Forschungsmethoden, zumal das interpretative Paradigma, zumindest in der Erziehungswissenschaft und der (Kindheits-)Soziologie längst zum anerkannten Bestandteil des Methodenkanons gehörte. Dennoch waren empirische Studien, in denen mit qualitativen Methoden gearbeitet wurde, in den ersten Jahrgängen der Frühen Bildung kaum vertreten. Dass es trotz einer anderen wissenschaftstheoretischen Fundierung der (Entwicklungs-)Psychologie und einer starken quantitativen Schwerpunktsetzung der empirischen Bildungsforschung dennoch gelungen ist, den Anteil von Beiträgen, die auf qualitativer Forschung beruhen, im Zeitraum von 2015 bis heute auf immerhin ein Viertel der Beiträge anzuheben, kann als Erfolg gewertet werden: 
Viele forschende Kolleg_innen haben bewiesen, dass ihre Forschung voraussetzungsvollen Gütekriterien und hohen Standards genügt und wichtige Erkenntnisse zu generieren vermag, die sowohl wissenschaftlich als auch für die Fachpraxis von großer Bedeutung sind. Erreicht wurde, dass die qualitativen Methoden in der frühpädagogischen Forschung sichtbarer geworden sind und stärker den Diskurs mitbestimmen, als dies vorher der Fall war. Mit einer Etablierung von Forschungszugängen im Wissenschaftsbetrieb durch eine Zeitschrift ist immer auch ein gewisser Standardisierungsdruck verbunden. Ein Blick in die qualitativen Beiträge, die in den letzten Jahren in der Frühen Bildung erschienen sind, zeigt allerdings, dass Kreativität, Offenheit und Innovationskraft qualitativer Forschungszugänge, die untrennbar mit ihrer theoretischen (interpretativen) Fundierung verbunden sind, erhalten werden konnten.

Den Anspruch der Frühen Bildung, sowohl pädagogische als auch (entwicklungs-)psychologische Perspektiven abzubilden, die Auswahl der Beiträge nicht an ihren methodologisch-methodischen Zugängen, sondern an deren Qualität und der Passung von Methodik und Fragestellung festzumachen und sich sowohl an die scientific community als auch an fachpraktische Akteure zu wenden, halten wir nach wie vor für richtig und innovativ. Gleichwohl sind dies auch Spannungsfelder, die das Interesse an der Zeitschrift und ihre Reichweite, anders als anfänglich erwartet, nicht nur stärken, sondern auch schwächen. Eine Herausforderung der nächsten Dekade wird daher darin bestehen, die Frühe Bildung für eine noch breitere fachlich affine Leserschaft zugänglich und attraktiv zu machen, und zwar, ohne Abstriche bei der wissenschaftlichen Qualität in Kauf zu nehmen. Schon jetzt sind Autor_innen auf- gefordert, eine Transferperspektive einzunehmen und sich auch zu den Implikationen ihrer Studienergebnisse für die pädagogische (Bildungs-)Praxis zu äußern - dies sollte beibehalten werden und ließe sich durch innovative Formate, wie bspw. eine regelmäßige Einordnung der wissenschaftlichen wie praktischen Relevanz der Beiträge eines Schwerpunktheftes noch fester verankern.

Eine weitere Entwicklungsperspektive ist es, der sich zunehmend etablierenden Mixed-Methods-Forschung Publikationsformate anzubieten, die ihren Besonderheiten besser gerecht werden, was u. a. eine Überprüfung der bislang üblichen Umfangs- und Gliederungsvorgaben notwendig machen würde mit dem Ziel, eine neue Balance zwischen Standardisierung und Flexibilität zu finden.

Schließlich muss es in den nächsten zehn Jahren darum gehen, noch viel stärker als bisher sowohl gestandene Forscherpersönlichkeiten als auch den exzellenten wissenschaftlichen Nachwuchs in die Gestaltung und Weiterentwicklung der Zeitschrift einzubinden - sei es als Autor_in, als Gutachter_in, in der Funktion als Beiratsmitglied oder Mitherausgeber in. Dies wird nicht nur den Bekanntheitsund Verbreitungsgrad der Zeitschrift steigern, sondern maßgeblich zur Qualitätssicherung im Sinne wissenschaftlicher Perspektivenvielfalt und Diskursivität beitragen.

Fabienne Becker-Stoll Klaus Fröhlich-Gildhoff Marcus Hasselhorn Iris Nentwig-Gesemann Franz Petermann $(t)$ Hans-Günther Roßbach Wolfgang Schneider Susanne Viernickel 